\title{
Une lecture nécessairement politique du phénomène « Kirdi » au Nord du Cameroun
}

\author{
Par Houli Fendjongue, Ngaoundéré
}

L'émergence de la question kirdi dans la vague des processus démocratiques actuels pose d'emblée dans le champ politique camerounais les enjeux politiques de la démographie. Celle-ci renvoie à la dynamique identitaire qui irrigue le système politique local et national avec pour principaux acteurs les investisseurs et autres entrepreneurs ethno-politiques qui, au regard de l'importance légitimante de la capitalisation du suffrage retrouvé, instrumentalisent les référents primaires pour se positionner sur les différentes échelles de l'échiquier politique. Dans la partie septentrionale du pays, le phénomène «kirdi» dont on a l'impression qu'il n y en a pas un est une réalité. Cela est dû à cause, d'une part de l'image monolithique présentée, entretenue et gardée par les uns et les autres à propos de cette région où tout le monde est considéré comme «Haoussa ${ }^{1}$ » et d'autre part par l'hostilité de la politique gouvernementale à l'expression et à l'affirmation des identités. Cette réalité qui avait été occultée pendant longtemps est aujourd'hui théorisée.

Aux yeux des analystes, le phénomène «kirdi » continue de poser problème. Deux choses sont certaines. D'une part, les populations dites «kirdi » sont présentes au Nord-Cameroun depuis des milliers d'année. Elles ont d'autre part, entre elles, des liens sur le double plan anthropologique et religieux. Sociétés de tradition paysanne, les «Kirdi », anciens dans leur terroir, y ont cumulé leur croît démographique au cours des âges. Le repli à l'époque des conquêtes peul a pu le renforcer encore. D'où les densités fortes de ces populations, voir très fortes dans les massifs montagneux et les plaines marécageuses de la région septentrionale du Cameroun. Ce sont ces accumulations démographiques, face à des potentialités limitées du milieu, qui expliquent, entre autres, au Xxème siècles les grandes migrations de travail et de survie du Nord vers le Sud.

Par le phénomène «kirdi », il faut comprendre le processus de construction d'une identité ethnique dont les éléments constitutifs proviennent des représentations historiques et contemporaines. En d'autres termes, c'est un mouvement visant à fondre dans un tout une pluralité de groupes sociaux dont le dénominateur commun originel reste la non apparte-

1

Le terme « Haoussa », aux yeux des Camerounais vivant dans la partie méridionale, s'appliquait à toutes les populations de ce qu'on appelait le Nord-Cameroun ( Adamaoua, Nord, Extrême-Nord ) au mépris de la diversité ethnique, linguistique, confessionnelle, etc. caractéristique substantielle de cette région du Cameroun. 
nance à la religion musulmane. Dans le même ordre d'idées, ce concept, bien que recouvrant une réalité incontestable, sert davantage d'outil opératoire d'analyse de ces rapports dans toutes leurs formes qu'un ensemble de populations entretient avec le système national global.

L'ampleur qu'a prise aujourd'hui le phénomène « kirdi », conceptualisé sous la dénomination D.C.K.(Dynamique Culturelle Kirdi), est à mettre à l'actif de la démocratisation amorcée au Cameroun depuis 1990, processus marqué par le relâchement des ressorts juridiques autoritaristes sur lesquels reposait l'édifice institutionnel du pays depuis 1960. Et le retour de la question renouvelle d'emblée dans le champ politique camerounais les enjeux de la supériorité numérique de cette communauté qu'on désigne par cet ethnonyme consacré. Aussi assiste-t-on ici à un cumul d'une identité contradictoire exprimée par une majorité démographique et une minorité politique « kirdi ».

Cependant, comment est né le phénomène kirdi comme problème politique ? Qu'est-ce qui fait sa «politisation» dans ce contexte précis de dé-construction et d'évolution de la vie socio-politique camerounaise comme on pouvait dire par exemple qu'est-ce qui fait la scientificité d'un concept ? Comment un concept colonial collé à des groupes sociaux épars est-il parvenu à polariser l'attention des acteurs politiques, des investisseurs identitaires, voire une mobilisation de la part de ceux qui sont désignés comme tels ? Bref, en quoi ce dispositif primordial, construction de nature stratégique, est susceptible de constituer un nœud des rivalités politiques au Cameroun ? Tels sont les axes de questionnement en état de servir de bien fondé à un débat sur cette question qui n'est plus un tabou, débat ou réflexion constructive pour la cohérence nationale et dont les conditions semblent bien réunies par ailleurs.

La réponse à toutes ces préoccupations de nature interrogative nous amène à définir d'une part ce concept (I) avant de dégager d'autre part la dimension véritablement politique de ce phénomène (II).

\section{Le contenu du concept « kirdi » : la double dimension ethnique intensive et extensive}

Aux fins d'analyse qui se veut profonde par rapport à un ensemble de groupes sociaux dénommés « kirdi » au nord du Cameroun, concept à forte prétention holiste au regard de l'hétérogénéité de cette composante nationale et de la diversité des modes de perception qui la caractérise, il convient de décrypter celui-ci sous la double dimension anthropologique (A) et sociologique (B). Ce qui permettra d'avoir une idée plus exacte de cette réalité. 
A. La conception primaire de la notion « kirdi » : la définition extensive de ce concept

D'emblée, il faut relever qu'il apparaît difficile dans le cadre de ce travail de recenser toutes les composantes de cette notion globalisante. Autrement dit, cette définition met en relief le pluralisme de la globalité « kirdi ». C'est le lieu ici de rendre un vibrant hommage au fructueux et ardu travail fourni par Jean-Baptiste Baskouda ${ }^{2}$, même comme celui-ci n'est qu'un ensemble d'ethnies compilées brutalement et présentées sous forme d'un document. Mais il a tout le mérite d'un travail de pionnier en la matière. Avec lui, un certain nombre d'auteurs ${ }^{3}$ et l'ouverture politique aidant, la question kirdi est belle et bien inscrite dans l'espace discursif camerounais.

Ce qui importe ici, c'est de savoir ce qui fait la «kirdité » de toutes ces entités sociales aujourd'hui intégrées dans ce groupe englobant. Qu'est-ce qui fait anthropologiquement l'unicité de cette pluralité septentrionale induisant une perception comme tel par toutes les consciences, internes et externes, du «dehors » comme du «dedans » ${ }^{4}$ ? Bref, qu'est-ce qui distille la «kirdité » comme scientificité d'un argument ou d'un concept ? Ou encore, le phénomène «kirdi » est-il une réalité ou une abstraction forgée pour des exercices purement intellectuels ? Les « kirdi » ont-ils la conscience de leur identité ?

L'existence ou non d'une ethnie ou d'un groupe social dit «kirdi » a alimenté les débats ces derniers temps ${ }^{5}$ avec le réveil des mouvements identitaires engendrés par la libéralisation des systèmes politiques amorcée depuis 1990 sous les tropiques en général et au Cameroun en particulier. Cependant, c'est un problème marqué du sceau de l'ancienneté mais qui reste intéressant et d'actualité et notre préoccupation dans le cadre de ce travail ne consiste pas à proposer une quelconque solution ni à prendre position pour les partisans de telle ou telle thèse mais de tenter de dégager les éléments qui paraissent assez objectifs pour cerner cette réalité aux contours controversés.

Ceci étant, les populations qui répondent de l'ethnonyme «kirdi » sont de manière globale toutes les tribus non musulmanes qui peuplent le Nord du Cameroun ou qui y sont originaires. Cela veut dire que ces gens se définissent religieusement en opposition à un autre

Jean-Baptiste Baskouda, Kirdi est mon nom, Yaoundé, Imprimerie St-Paul, 1993, 121 p. Signalons aussi que l'auteur de cet opuscule est le promoteur engagé du mouvement kirdi, c'est l'idéologue de la « kirditude ».

On peut citer entre autres P.Bigombé Logo, « Changement politique et dynamique d'instrumentalisation de l'ethnicité kirdi » in L. Sindjoun, (ed.), La révolution passive au Cameroun, 1999, pp : 231-268.

Nous empruntons ces expressions à G. Balandier, Sens et puissance, Paris, 1986, 334 p.

Nous pouvons appuyer notre argumentation sur ce malentendu qui oppose sur le plan strictement scientifique Reyang Bloum, Daniel Souloukna et Jean-Baptiste Baskouda. Voir à propos : L'œil du Sahel $n^{\circ} 33$ du 26 juin au 10 juillet 2000, pp : 6-11. 
groupe que sont les musulmans ${ }^{6}$ c'est-à-dire ceux qui ont embrassé l'Islam comme religion. Autrement dit, le «kirdi » est celui que la littérature musulmane désigne sous l'appellation de «kado» avec son pluriel «habé», qui signifie tout simplement mécréant, païen, paganiste, impur, vivant une convivialité cosmique régie par un attachement aux esprits surnaturels ; et même islamisée, toute personne de confession chrétienne n'est pas moins épargnée de cette épithète péjorative. Il s'agit d'une création négative de l'autre. Considéré sous cet angle, est «kirdi » tout être humain ne pratiquant pas la religion musulmane et habitant un espace compris entre les trois provinces composant le Nord-Cameroun ${ }^{7}$.

C'est donc le facteur religieux qui fait office de joker dans la définition des populations «kirdi » du nord du Cameroun. Rentrent dans cette catégorie ${ }^{8}:$ les Toupouri, les Massa, les Moundang, les Guiziga, les Mafa ou Matakam, les Mofou, les Dii, les Guidar, les Baya, les Zoulgo, les Mada, les Namchi, les Fali, les Tikar, les Mousseye etc. Dans cette perspective et par extension, même un Ewondo, un Bassa, un Bamiléké, un Douala ou un Bakwéri n'est pas moins «kirdi »s'il remplit les deux conditions relatives à la religion et à l'espace géographique.

Cette dichotomie peul-kirdi est supposée sous-tendre ce qu'il convient d'appeler la question kirdi qui, jusqu'en 1924, signifiait que les groupes non islamisés sont un problème pour la simple raison qu'ils résistaient aussi bien à l'autorité coloniale qu'à celle des souverains peul ou Laamibe ${ }^{9}$. Aussi à partir de cette date, la réorientation de la politique indigène de la France au Nord-Cameroun ${ }^{10}$ s'opère au détriment des Peul perçus comme les bourreaux de ces peuples «kirdi ». Pour l'administration coloniale soucieuse et désireuse de ramener la paix dans la région par la voie moins violente, pacifique grâce à la technique d'apprivoisement, le problème kirdi s'analyse en termes de refus de l'autorité des chefs musulmans occasionnant régulièrement des accrochages entre ces derniers numériquement minoritaires et les premiers occupants de la région plus nombreux. En l'absence des sta-

Constituent le groupe musulman ou islamo - peul au Nord - Cameroun : les Peul ou Foulbé, les Haoussa, les Mandara, les Arabes Choa, les Kotoko, les Kanouri ou Bornouan. Ces populations ont embrassé l'islam comme religion.

Il s'agit des provinces de l'Adamaoua, du Nord et de l'Extrême-Nord conformément au découpage territorial opéré par le décret présidentiel du 22 août 1983.

8 Il serait utile de se reporter à l'ouvrage de J.-B. Baskouda, op. cit.

Laamibé est le pluriel de laamido. Ce dernier se définit comme un centre de production du politique défini non seulement comme rapport de commandement et d'obéissance, mais comme accumulation de l'influence englobant toutes formes de domination, y compris celle économique. Voir à propos les fructueuses investigations de Motazé Akam, Le défi paysan en Afrique. Le Laamido et le paysan dans le Nord - Cameroun, Paris 1990.

10

Sur la politique indigène de la France au Nord - Cameroun, voir utilement D. Abwa, Commissaires et hauts commissaires de la France au Cameroun (1916-1960), Yaoundé, Presses Universitaires de Yaoundé et Presses de l'Université Catholique d'Afrique Centrale, 1998. 
tistiques officielles, ce groupe n'est pas moins considéré dans les analyses ethnisantes comme le plus démographiquement important par rapport aux islamo-peul ${ }^{11}$. Ce qui fait ressortir clairement au plan régional un problème de déséquilibre politico-démographique alimentant et servant de support actuellement aux revendications politiques des populations dites « kirdi » de cette aire géographique du Cameroun. L'enjeu s'articule ainsi autour de la traduction politique du poids numérique « kirdi ».

Outre la religion non musulmane qui apparaît ici comme l'élément fédérateur fondamental des populations «kirdi », certaines normes participant du fonctionnement de leurs sociétés les rapprochent davantage. Cependant, il faut relever que l'arrivée dans la région des pasteurs/nomades Peul ou Foulbé joua un rôle fondamental sur le plan religieux dans la mesure où certains groupes comme les Guiziga, les Moundang, les Fali, les Mousgoum, etc. ont été plus ou moins convertis à l'islam sans que cela atteigne pour autant leur fonds religieux animiste. Aussi sur le plan culturel, les pasteurs foulbé ont réussi à faire répandre leur langue, à savoir le «foulfouldé », dans toute la région au point où, de nos jours, celuici sert de véhicule de communication entre tous les groupes ethniques nord-camerounais. Toutes les populations « kirdi » sont à l'heure actuelle «foulbéiphones » 12 à l'exception de quelques rares personnes réfugiées dans les grottes des montagnes inaccessibles et qui continuent à développer des stratégies d'obstruction à toute tentative de modernisation.

Ainsi sur le plan économique par exemple, les structures traditionnelles des populations « kirdi » présentent un mode de production lignager qui est le fondement essentiel des mécanismes de fonctionnement économique. Le mode de production configure des rapports sociaux de production régis par des inégalités statutaires (classes d'âge, sexe, etc.) et repose sur un modèle de production simple consacrant l'économie de subsistance, sans que l'on aboutisse à des véritables clivages sociaux connus présentement par ces sociétés depuis leur insertion et leur mouvance dans le réseau complexe du capitalisme international.

Sur le plan politique, l'autorité et le pouvoir restent ouverts et disséminés à travers des unités territoriales réduites consacrant le caractère résiduel de l'organisation politique et administrative païenne. On n'y trouve nulle part l'exemple du laamido rencontré dans la tradition politique islamique, preuve que la stratification sociale n'est pas rigidement bipolaire comme dans les sociétés musulmanes. L'exercice du pouvoir et de l'autorité est fait de

A propos de la supériorité numérique des non musulmans sur les musulmans, les analyses sont constantes. Voir à propos : les statistiques fournies par A. Beauvilain, Nord - Cameroun, crises et peuplement, Rouen, 1989, M. Prouzet, Le Cameroun, Paris 1974. Se référer aussi à certains

12 rapports : ANY, APA11618, Région du Nord - Cameroun, rapport annuel d'ensemble, 1948.

Nous entendons par ce terme tous ceux qui parlent le dialecte foulbé ou peul à la suite du phénomène d'emprunt tout comme un Nègre qui cause la langue française et/ou anglaise. 
structures parallèles ${ }^{13}$. Ils n'impliquent pas ici nécessairement l'existence d'un Etat, ce qui ne remet nullement en cause la théorie scientifiquement établie par Pierre Clastres ${ }^{14}$ selon laquelle une société sans Etat n'a pas pour corollaire une société sans pouvoir politique. Dans le présent cas, la régulation politique englobe le visible et l'invisible, le réel et l'imaginaire, enfin le concret et le symbolique ${ }^{15}$.

En dehors de ces quelques traits fédérateurs, les populations dites «kirdi » sont traversées par des différences fondamentales. Elles sont par essence hétérogène surtout sur le double plan linguistique et géographique. En d'autres termes, ces composantes « kirdi » parlent des dialectes distincts les uns des autres, habitent des espaces hétérogènes en ce sens que certaines vivent dans les massifs montagneux, d'autres dans les plaines. Ces grandes différences peuvent amener l'analyste à parler d'une hérésie sociale quand il faut considérer ces gens comme une seule entité ethnique.

Si des faits historiques et anthropologiques permettent d'accepter la réalité d'une entité susceptible d'être appelée «kirdi », cela ne semble pas suffisant pour affirmer de manière péremptoire qu'il existe une conscience identitaire « kirdi » intentionnellement constituée. Autrement dit, que pensent ces populations de leur identité ? Cette dernière est-elle contraignante et extérieure à ces gens ?

Sans entrer dans un débat apparemment sans issue, tout porte à croire que l'identité « kirdi » reste problématique en ce sens que son ancrage dans les consciences reste symbolique et superficiel. Sa réalité pratique n'est pas perceptible et par conséquent celle-ci renvoie à une construction intellectuelle et politique visant à légitimer des situations précises. C'est dire qu'en tout état de cause, il est à remarquer que loin de remettre en cause la réalité du phénomène «kirdi », il y a lieu de reconnaître qu'il reste pour l'essentiel une affaire d'élites politiques, surtout urbaines, les cadets sociaux, la grande masse paysanne et l'immense petit peuple misérable étant très désintéressés et déconnectés de ces débats, de ses tenants, aboutissants et enjeux, surtout avec l'analphabétisme ambiant.

En fait, la question qui porte sur l'existence ou non de la conscience ethnique « kirdi » commande tout le débat historique et actuel de la thématique. Et elle a tout son mérite dans

Motazé Akam, «Changements socio-économiques et maintenance sociale chez les kirdi du Cameroun Septentrional », in Revue Science et Technique, n 3-4 , 1989, pp. 125-138.

14

P. Clastres, La société contre l'Etat, Paris 1974.

15

Pour une intelligibilité des modes de fonctionnement des sociétés «kirdi », voir à titre indicatif : D.I. Garine, Les Massa du Cameroun, vie économique et sociale, Paris 1961, 250 p ; J. Guillard, Golompoui : analyse de modernisation d'un village du Nord - Cameroun, Paris 1965; G. Pontié, Les Guiziga du Cameroun Septentrional, Paris 1973; l'ouvrage collectif : Nord - Cameroun, des Hommes. Une région, Paris 1984 ; J.-F. Vincent, Princes montagnards du Nord - Cameroun, Paris 1991. 
la mesure où le terme «kirdi » est chargé d'une bonne dose d'éléments péjoratifs, de mépris aux yeux des destinataires. C'est pourquoi celui-ci fait l'objet d'une naissance pour autrui. Les «non croyants » des plaines, par exemple, estiment que ce terme ne s'applique qu'aux seuls habitants des montagnes ${ }^{16}$, plus particulièrement aux populations dites «Mofou» alors que ces derniers rejettent aujourd'hui celui-ci se voulant seulement «gens de montagne » comme l'a remarqué Jeanne-Françoise Vincent ${ }^{17}$. Pourquoi un terme, fut-il utile ou très important, suscite-t-il autant de passion et de controverses ?

Si ce concept avait été avancé en toute innocence, ce qui ne semble d'ailleurs pas être le cas, pour désigner des groupes ethniques avec lesquels on ne partage pas les mêmes convictions religieuses ${ }^{18}$, compte tenu de sa charge méprisante, il a du mal à s'enraciner dans les milieux cibles. La contestation a été exacerbée et ravivée à cause des multiples brimades et traitements ignobles et indignes subis par les «mécréants » des plaines et des montagnes sous le régime du président Ahidjo. C'est peut-être en raison de ces réactions que le terme «kirdi » est en train, du moins de disparaître du vocabulaire administratif, sinon de changer de site, donc de champ politique.

Tout compte fait, nul ne doute que le mot « kirdi » est de plus en plus contesté à cause de sa charge péjorative alors que d'après sa définition initiale, il reflète les réalités culturelles des dites populations ; certainement à cause des déformations subies par ce concept au fil du temps. La «kirtitude» se vit plus par le «haut» que par le «bas », c'est-à-dire sans les vrais «kirdi ». Cela confirme cette réalité que l'on vit aujourd'hui avec la juxtaposition desdites sociétés sans aucune prétention d'unité et multipliant leurs propres fêtes culturelles à l'instar du festival Massa, de la fête du coq chez les Toupouri, de la fête de Muzgla chez les Guidar, le Maraï chez les Mafa, etc.

$\mathrm{Au}$ fur et à mesure que ce terme se vide de son contenu ethnologique, il se charge d'une bonne dose politique dont beaucoup d'ingrédients se trouvent dans sa définition sociologique.

Source : enquête.

17

J.-F. Vincent, op.cit., p. 41.

18 Le terme serait employé par le major Denham, premier voyageur à parcourir la région. Il n'imitait que ses compagnons Bornouan et Arabes. Les militaires français l'emploient dès leurs premières tournées dans la région en 1918 et les premiers administrateurs l'adoptent aussi. Il a été longtemps employé par les différentes administrations camerounaises (cf. Le timbre à $60 \mathrm{f}$ cfa en 1979, «Village Kirdi ». Voir à propos de tout cela, J.-F. Vincent, op. cit. p. 41. 


\section{B. La conception sociologique du terme «kirdi » : l'édification ethnique intensive}

Inversement à la diversité ethnique dégagée par les ethnologues, au pluralisme dialectal mis en relief par les linguistes se substitue l'unicité ethnique «kirdi » de facture politique mise au point par les sociologues et les politistes. Celle-ci trouve son fondement, sa justification et sa cristallisation autour de la domination islamo-peul et la dépendance desdites populations.

Ainsi, comme le disait si bien Max Weber qu' «il n'y a pas de domination acceptée sans mythes pour la soutenir, la légitimer et la faire respecter ${ }^{19}$, le concept de « kirdi », sans l'intention de nier sa charge ethnologique, semble avoir été forgé par les coreligionnaires de Mohamet pour un but qu'il n'a pas moins atteint : déstabiliser psychologiquement les autochtones et se passer aux yeux des premiers voyageurs occidentaux pour les légitimes dépositaires de l'autorité de cet espace régional encore trouble. Jean Ziegler a d'ailleurs constaté avec rigueur dans ce sens qu' «il n'y a pas de prise de pouvoir politique sans prise préalable du pouvoir culturel ${ }^{20}$.

En filigrane de la pensée de ces deux auteurs se dégage le phénomène de la domination qui semble être le critère sociologique le plus objectif pour la définition du concept de «kirdi ». En d'autres termes, saisir la domination comme élément participant de l'édification du vocable « kirdi » renvoie à l'appréhension desdites populations dans les rapports qui président au fonctionnement de la société dans laquelle elles vivent et en même temps faisant d'elles une seule ethnie au mépris de la diversité linguistique et anthropologique. Autrement dit, quelle place occupent-elles dans l'architecture de la société qui les englobe ou plus concrètement quelle position les populations «païennes » occupent dans le dispositif structurel de la société camerounaise.

En fait, le concept de «kirdi » se définit plus nettement, aussi bien par auto-perception que par exo-perception, par la dépendance dont ces populations sont objet par rapport au groupe islamo-peul. La situation de dominés constitue la variable fondamentale sur laquelle les «mécréants » du Nord-Cameroun se reconnaissent comme tel. Vécue au quotidien, la domination musulmane sur les non musulmans représente l'élément de cristallisation de la conscience ethnique «kirdi ». Ce qui importe présentement, c'est de savoir comment cela s'est ancré sociologiquement dans les représentations locales et nationales ?

Ainsi, sur le plan culturel, l'islamisation fut l'arme utilisée par les coreligionnaires de Mohamet pour exprimer leur supériorité sur les «habe » c'est-à-dire les «païens » en pro- 
cédant à leur conversion à la religion musulmane, usant de tous les moyens possibles et disponibles. Cette stratégie de destruction de la personnalité naturelle et d'acculturation de ces derniers eut des fortunes diverses ${ }^{21}$. Cependant l'objectif d'une telle entreprise était d'asseoir la domination musulmane par l'asservissement culturel des non musulmans. Charles Robert Dimi l'a bien observé avec pertinence lorsqu'il dit que «toute domination d'un peuple par un autre suppose, de la part du peuple dominant, une praxis aliénante. Celle-ci consiste dans le fait que le peuple dominant se projette sur le peuple dominé (il veut le façonner à son image) » 22 .

Dans l'optique de détruire l'univers symbolique des «kirdi », indice de la domination culturelle musulmane, une équivoque mérite d'être levée. En fait, l'observation profonde des sociétés païennes au nord du Cameroun laisse transparaître une forme d'islamisation de type utilitaire, voire instrumentalisée. Elle s'observe dans le milieu élitiste «kirdi », surtout chez les chefs traditionnels qui, en même temps ne croient pas à l'islam comme religion, mais récupèrent à leurs comptes personnels son idéologie avec tous ses avantages politiques et économiques. Ils s'en servent à des fins d'exploitation et de ponction de leurs sujets. Ce qui crée inévitablement des conflits au sein de la civilisation païenne.

Dans cette perspective, l'islam comme religion a servi à exprimer la domination du groupe islamo-peul sur les «non croyants » puisque certains se sont convertis en renonçant à leur culture d'origine devenant par là culturellement ambigus et fonctionnant aisément dans plus d'un registre ethnique. Ils se font, par exemple, musulmans tout en demeurant « impurs », l'inverse étant cependant rarissime. Ce qui est une réalité dans cette région.

En outre, ce phénomène entretient l'expansion de l'islam en milieu païen tout en camouflant la véritable moitié culturelle des animistes. Enfin, pour les élites «kirdi », l'islam est un enjeu qui acquiert le statut d'un mal nécessaire. Même s'il faut recourir à sa propre religion, le paraître musulman est prééminent. Tout paraît donc concourir à fonder l'hypothèse selon laquelle les lois du phénomène d'islamisation sont telles que même en refusant intérieurement l'islam, on s'accroche paradoxalement aux appétits matériels de la religion.

Au vu de ce qui précède, même si le « kirdi » instrumentalise la religion musulmane, ce que nous pouvons appeler ici une islamisation sans islam, il n'en demeure pas moins qu'il soit culturellement dominé et cela mérite aussi d'être analysé sur le plan économique. 1991. 
La dépendance économique est une variable repérable qui structure la définition de l'identité ethnique «païenne » au Nord-Cameroun. Elle résulte davantage de l'exploitation des paysanneries locales par la classe laamidale et de l'absence d'initiatives économiques de celles-ci. Le système est construit de manière que toute initiative « kirdi » semble vouée à l'échec car uni sous ce holisme, mais désuni sous toute forme d'initiative économique ou politique. Une pratique séculaire autorise le laamido à envoyer les paysans travailler dans ses champs de coton et de «muskwari », sorte de variété de mil local d'origine musulmane. Cette force de travail paysanne est exploitée gratuitement pour la production laamidale. La mise en auvre du mode de production tributaire consacre la domination économique musulmane sur les sujets - paysans. Economiquement dominés, ceux-ci ne peuvent plus échapper à la domination politique modulée par la religion islamique.

En fait, les mécanismes d'oppression économique des populations «kirdi », surtout leur segment paysan, sont nombreux et puriformes; ils n'obéissent à aucune réglementation dans la mesure où ils s'opèrent dans une partie du pays où l'arbitraire préside à la régulation des rapports entre les dominants et les dominés. C'est pourquoi il apparaît ici difficile de les répertorier et de les analyser en détail. Cela ne constitue nullement un obstacle insurmontable pour la connaissance des phénomènes qui ont sécrété les inégalités matérielles au sein des composantes ethniques septentrionales où les « kirdi » sont sans doute les acteurs du bas.

A cet effet, la domination «kirdi » résulte des procédures historiquement établies. Elles consistent en l'imposition en argent et l'instauration des cultures obligatoires dans le cadre de l'exploitation par la grande propriété capitaliste des moyens de production et de la force du travail. Certes, la grande propriété capitaliste n'a jamais existé en milieu nord camerounais sous la forme qu'elle a revêtue dans la région du Sud Ouest du Cameroun telle qu'analysée par Vianney Ombé Ndzana ${ }^{23}$, mais à mesure que le mode de production capitaliste s'est étendu, le système a pu, dans certaines limites, reproduire de lui-même les conditions matérielles et sociales d'exploitation.

Ceci étant, l'appauvrissement des paysans « kirdi » s'est fait à travers des entreprises capitalistes de production agricole et des trusts internationaux qui commercialisent la production rentière et agricole autochtone. Ceux-ci sont représentés ici par la $\operatorname{SODECOTON}^{24}$ et l'Office Céréalier ${ }^{25}$, bien que ces derniers aient subi une restructuration aujourd'hui. Mais ensemble, ils ont provoqué la destruction des unités de production parcellaires, lignagères 
et tribales, la transformation des rapports, bref, l'émergence d'autres formes des rapports sociaux fondés sur la verticalité où les paysans subissent une domination à trois niveaux : l'aristocratie féodale laamidale, l'Etat et la société internationale.

Si ces organismes avaient initialement des objectifs de développement en milieu rural, ils ont fini par avoir des mécanismes de fonctionnement de vraies entreprises capitalistes. Leurs évolutions respectives ont abouti à une stratification sociale en groupes antagoniques dans la mesure où par leurs actions dans la pratique du travail agricole, ils ont développé une bourgeoisie administrative composée des techno-bureaucrates d'une part, et une bourgeoisie rurale où se retrouvent les chefs islamisés d'autre part. Par conséquent ces deux organismes ont contribué à la marginalisation de la majorité des petits paysans «kirdi ». Leur développement s'est accompagné de la cristallisation des inégalités sociales en milieu rural et de la consolidation des rapports clientélistes entre les chefs islamisés et l'administration.

Au Cameroun, à travers l'agro-industrie, les mécanismes de fonctionnement de l'Etat opèrent une dé-paysannisation transformant le seigneur de la terre qu'est le paysan en simple ouvrier ou manœuvre. Ce solde sacrifiant la paysannerie sur laquelle repose le développement par la trilogie dominatrice, aboutissement des extorsions, des ponctions, des marginalisations et parcellisations paysannes, passe nécessairement par l'idéologie religieuse, donc islamique qui légitime toutes les échelles du système de domination.

Bref, économiquement, le « kirdi » au Nord-Cameroun est ce migrant qu'on rencontre dans les zones rurales de Pitoa, Bibémi, Touboro, Lagdo, Poli, etc. vendant sa force de travail pour sa survie et celle de sa famille. En ville, faute de qualification, il est pour la plupart veilleur de nuit, secteur informel où le droit social est absent ${ }^{26}$, et vivent dans les zones de relégation sociale où la sociologie urbaine nous permet de comprendre la structure dichotomique de domination et de subordination. Le dispositif des groupes sociaux met en relief les dominants musulmans et bureaucrates habitant le centre et les dominés «kirdi » résidant à la périphérie où ils disposent du monopole de ce que Nici Nelson appelle «l'industrie de divertissement ${ }^{27}$ qui englobe toutes les formes de prostitution, de la production de bière locale brassée illégalement et liant dans un même mouvement d'opposition à la police tous les protagonistes, du producteur au consommateur. C'est le cas des quartiers comme : Pont, Domayo, Houro-Tchédé, Pittowaré à Maroua ; Roumdé, Djamboutou, Basa à Garoua et enfin Joli Soir, Bourkina, Gada-Mabanga à Ngaoundéré.

C'est un secteur où il n'existe ni horaires contrôlés, ni rémunérations minimales, ni comptabilité légale, ni à fortiori syndicalisation.. world cities, New York 1979, pp. 283-302. 
Grosso modo, l'identité ethnique « kirdi » se définit sous le prisme de la non appartenance à la religion musulmane, de la domination culturelle et de la dépendance économique. Ce sont «les laissés-pour-compte ${ }^{28}$ de l'architecture de la société nord camerounaise. Ceuxci tentent aujourd'hui d'ériger leur poids démographique en ressource politique à la faveur de la démocratisation. Cela signifie que les données démographiques contiennent déjà un élément essentiel de compréhension de la tension qui a toujours régné autour de la question «kirdi » au Nord-Cameroun : les «foulbéiphones» ne sont pas des minorités politiques insignifiantes que l'Etat central pourrait facilement «oublier». Ce sont des masses démographiques considérables et de ce fait posent un problème à l'Etat tout comme les ethnies «Anglo-bami », « Béti », «Sawa », etc. selon le contexte politique qui prévaut ; c'est-à-dire en fonction de la position ou de l'appartenance « ethnique » du pouvoir suprême.

Du point de la stricte observation du réel social, la question kirdi, régulièrement évoquée et brandie par la classe politique lorsque la composition et/ou la recomposition des positions du pouvoir est en jeu aussi bien sur le plan local que national, n'est plus un mythe. Tel le problème actuel concernant la succession de l'ex-ministre d'Etat Sadou Daoudou, décédé en 2002 à Ngaoundéré, au poste de vice-président du RDPC, parti au pouvoir, par exemple. Cette succession représente un enjeu politique véritable et aigu, car ce choix peut être un indice d'orientation de la succession présidentielle, entre «kirdi » et musulmans ${ }^{29}$ et de moindre envergure entre élites de l'Adamaoua, du Nord et de l'Extrême-Nord reléguant au second plan le clivage confessionnel au profit du conflit régional.

Pour tout dire, il est permis de penser que si les « Kirdi » constituaient un ensemble spatial compact et non un chapelet discontinu et diversifié d'habitat, disposaient d'une communauté linguistique et non d'une pluralité de dialectes à chaque groupe, et mus par la recherche d'intérêts supérieurs de la communauté reléguant au second plan les considérations égoïstes, l'histoire lointaine et récente du moins du Cameroun, si non du Nord-Cameroun aurait été différente et qu'ils représenteraient aujourd'hui une puissante force politique réalisant ainsi une identité politique « kirdi » dans le contexte de démocratisation. que : Adjoudji Hamadjoda (musulman de la province de l'Adamaoua où était originaire le défunt ministre d'Etat), Yaya Marafa Hamidou ( musulman de la province du Nord ), Luc Ayang, Ali Amadou, Djibril Cavaye Yegué et Dahirou Sali (élites de la province de l'Extrême-Nord, musulmans à l'exception de Luc Ayang qui est kirdi). 


\section{Le phénomène « kirdi » dans l'optique de la démocratisation : le positionnement politique comme facteur de légitimation d'une identité}

Dans l'optique de l'ouverture politique, les acteurs politiques individuels ou collectifs, tentent d'élaborer des stratégies d'investissement des réseaux du pouvoir, gage de leur visibilité au sein du système politique rendu ouvert, souple et accessible. Et dans ce cocktail de canaux d'accès à l'Etat, voire de conquête de reconnaissance sociale ou plus précisément de mobilité sociale, on y trouve: des entreprises politiques comme les partis, les structures manifestement de soutien au pouvoir en place comme la PRESBY ou Jeunesse de Biya, les organisations d'influence du pouvoir dont l'objectif réel ou virtuel est la promotion de leurs membres, les regroupements de médiation avec le pouvoir comme les associations des élites ou des ressortissants que l'on rencontre un peu partout, les associations culturelles ou identitaires d'animation politique, la liste est loin d être exhaustive.

Quelque soit l'épithète qu'on puisse coller à ces types de structures, toutes laissent transparaître la volonté de participation et de gestion de l'espace public. Elles sont mues par le désir d'accéder au champ politique, soit directement ou indirectement. Elles entendent conquérir les positions de pouvoir et sont toutes politiques ou à connotation politique. Et le phénomène «kirdi » n'échappe pas à cette règle presque universelle. Sa dimension politique saute à l'œil nu après une analyse sociologique de ses promoteurs (A) et à cause de sa thématique mobilisatrice (B).

\section{A. L La sociologie des acteurs de la "kirditude», une mosaïque d'élites politiques comme entrepreneurs culturels : la Dynamique Culturelle Kirdi (D.C.K.)}

En principe, l'acteur se reconnaît dans le théâtre ou le cinéma. Pris sous cet angle, il est véritablement acteur social en ce sens qu'il est imbriqué dans un tissu de rapports sociaux en vue d'une action dans la vie pratique. La différence entre un acteur de cinéma et un acteur politique se situe d'abord au niveau où l'acteur de cinéma et de théâtre joue un rôle en termes de personnage de l'acteur politique. Cependant la similitude entre les deux catégories d'acteur est que, forcément, les deux ont un rôle déterminé. La notion de rôle implique nécessairement celle d'acteur. L'acteur d'un roman par exemple joue le rôle d'un acteur politique à travers le personnage qu'il incarne. Alors que le rôle du premier est fictif, celui du second est réel.

Il n'y a pas d'acteurs politiques sans pratique politique qui les imbrique dans un système de relations. Les auteurs comme Guy Rocher ${ }^{30}$ et Alain Touraine ${ }^{31}$ ont mené des études qui se 
sont intéressées sociologiquement aux notions d'acteur et de l'action. La caractéristique fondamentale des acteurs politiques reste leur nécessaire lien avec un groupe, avec une collectivité en vue d'une action. C'est «un ou des individus engagés dans une action, et donc observables a travers un champ déterminé » comme l'a remarqué Motazé $\mathrm{Akam}^{32}$. Les acteurs sociaux, donc politiques, peuvent paraître soit en termes d'individus insérés dans des relations sociales, soit en termes de classes que Touraine ${ }^{33}$ définit comme des acteurs conflictuels de l'historicité d'une société quelconque.

La Dynamique Culturelle Kirdi est une association à caractère culturel selon les dispositions de son règlement intérieur. Elle est née au début de la décennie 1990 suite à la libéralisation de la société camerounaise. Ses fondateurs historiques furent Jean-Baptiste Baskouda, Wapou Jacob et Tikela Kemone ${ }^{34}$. Cette structure prônait à ses débuts la revalorisation des cultures ancestrales des peuples dits «kirdi » sans objectifs manifestes de sombrer dans les luttes englobantes de positionnement politique. Nous y reviendrons dans les lignes qui suivent dans la mesure où la D.C.K. et la « kirditude » semblent avoir la même signification.

Ceci étant, nous définissons les acteurs sociaux de la «kirditude» ou de la D.C.K. comme des acteurs agissant en groupe au nom des populations dites « kirdi » dans le champ politique camerounais sous le prétexte de la promotion culturelle de ces dernières. Cette entreprise ne peut être perçue que sous l'angle politique compte tenu de la position de ces acteurs sur l'échiquier national et de la nature du combat qu'ils mènent, combat dont la rentabilité reste liée à la visibilité des uns et des autres sans répercussions directes sur les véritables destinataires de la lutte.

Le phénomène «kirdi » est le champ d'observation par excellence d'acteurs politiques impliqués dans un processus d'émancipation culturelle où le regard critique permet de faire une lecture politique d'une entreprise à partir de ses acteurs. En d'autres termes, peut-on lire autrement que politique l'entreprise de redécouverte et de revalorisation du patrimoine culturel «kirdi » altéré par les colonisations occidental et peul menée par des acteurs du système camerounais ? Est-ce que l'élite politique «kirdi» est culturellement qualifiée pour mener à bien une politique de promotion des consciences sclérosées par des mœurs importées pour elle et pour les siens ? En d'autres mots, peut-elle s'ériger en entrepreneur culturel véritable et valable sans utiliser cela à des fins de positionnement politique ? Une sociologie des acteurs de la «kirditude » s’impose ici pour démasquer les choses. 
Sans prétendre faire l'inventaire exhaustif de toutes les élites « kirdi », ce qui ne semble pas être une entreprise aussi facile, nous nous contentons de nous en tenir aux personnalités les plus influentes et qui semblent s'intéresser véritablement à la « chose kirdi ».

Ainsi, dans la panoplie de ces «entrepreneurs culturels » ou animateurs de la D.C.K., l'on peut citer entre autres : Ayang Luc, ancien ministre de l'Elevage, des Pêches et des Industries Animales, ancien Premier Ministre par intérim, actuel Président du Conseil Economique et Social, membre du Comité Central et du Bureau Politique du Rassemblement Démocratique du Peuple Camerounais, parti politique au pouvoir; Jean-Baptiste Baskouda, ancien Directeur Adjoint du Cabinet Civil de la Présidence de la République et actuel Secrétaire Général du ministère de l'Agriculture ; Zacharie Pérevet, actuel ministre de la Recherche Scientifique et Technique ; Denis Oumarou, Secrétaire d'Etat à la Jeunesse et Sports ; Tikela Kemone, ancien Secrétaire d'Etat à l'Agriculture et actuel Conseiller Spécial du Chef de l'Etat ; François-Xavier Darwé, ancien Directeur de l'Administration Générale au ministère de l'Elevage; Djokna Damsala, contrôleur provincial des finances de la province du Nord àGaroua ; feu Dr Pierre Lingwé, ancien Chef de la Division Administrative à l'Ecole Nationale d'Administration et de Magistrature ; Zacharie Ngniman, ancien député à l'Assemblée Nationale et journaliste au ministère de l'Information; Aoudou Bello, actuel Président de l'association des populations Baya dénommée MOINAM et contrôleur financier auprès de l'Université de Dschang ; etc.

L'absence des personnalités «kirdi » comme Dakolé Daïssala, Toupouri, Ministre des Transports et Président du Mouvement pour la Défense de la République, parti d'opposition ; Hélé Pierre, Moundang, transfuge de l'Union Nationale pour le Progrès et la Démocratie et actuel Ministre de la Faune et la Protection de la Nature, etc. est très significative du point de vue de la duplicité de l'idéologie originelle du phénomène «kirdi » et même de la précarité de sa construction politique. Cette absence participe aussi du fait que, la mobilisation politique des clivages ethniques et régionaux en tant qu'action organisée, subit le poids des contraintes et l'indétermination des contingences au point de renvoyer en fin de compte à des bricolages aléatoires comme l'a souligné Friedberg ${ }^{35}$. Expliquant davantage la construction fragmentée de l'ethnicité, Luc Sindjoun rappelle «que ce qu'on appelle la mobilisation politique de l'ethnie bamiléké est aussi le fait des opposants au régime que celui de ses partisans ${ }^{36}$. Tout cela revient à dire qu'il n'y a pas une manipulation totalement réussie de l'ethnicité par les élites.

Au vu des personnalités ci-dessus citées considérées comme les promoteurs de la DCK, tout laisse à penser qu'il s'agit des personnes tributaires du pouvoir de nomination que le 
Chef de l'Etat, président du RDPC, détient pratiquement seul. Ce qui laisse aussi transparaître la subordination de celles-là à celui-ci. Autrement dit, dans un tel contexte, quelle crédibilité accorder à un projet congénitalement si vulnérable ? La réponse à cette question est commandée par l'analyse de la thématique mobilisatrice du mouvement culturel « kirdi ».

\section{B. La marginalisation comme élément mobilisateur et stratégique de positionnement politique}

D'emblée, convient-il de distinguer l'ethnicité politique «kirdi» des autres types d'exploitation des référents identitaires connus ailleurs. Autrement dit, pourquoi la marginalité politique des « kirdi » au Nord-Cameroun, qui est un phénomène difficile à démentir, n'a pas ou pas encore conduit à la polarisation des exclus contre les dominants et à la formation progressive des sentiments d'hostilité des uns contre les autres de type Hutu / Tutsi telle que analysée par Dominique Darbon ${ }^{37}$.

En fait, l'ethnicité septentrionale «kirdi » telle que vécue aujourd'hui est le prototype de ce que nous pouvons qualifier d'ethnicité contextuelle, c'est-à-dire un produit de situations données. Elle renvoie à des situations où les conditions socio-économiques qui prévalent sont à l'origine des relations conflictuelles. Celles-ci ne se sont jamais cristallisées autour d'antagonismes entre musulmans dominants et «kirdi » dominés transformant cet état des choses en enjeu capital de lutte frontale et meurtrière ${ }^{38}$, hormis quelques scènes de violences sporadiques observées ça et là entre pasteurs et agriculteurs, etc.

En outre, l'avènement du président Ahidjo, musulman, au pouvoir en1960 a enfoui dans les subconscients toutes velléités d'expression d'une quelconque ethnicité, fut-elle «kirdi », avec l'ambiance d'une poly-ethnicisation aidant, c'est-à-dire que chaque situation, chaque geste dégage une forme d'ethnicité spécifique à ce moment précis. Ce qui signifie qu'au sein du monde « kirdi », il existe d'autres formes de particularités qui produisent à leur tour l'ethnicité. Tout cela constitue d'écueils obstruant les mobilisations frontales. Et même aujourd'hui avec l'introduction du pluralisme politique où il y a risque de renaissance des passions ethnicistes, tout est fonction du comportement de la classe dirigeante, surtout de sa discipline car ses tendances hégémoniques peuvent prévoir les manifestations conflictuelles

D. Darbon, «De l'ethnie à l'ethnisme, réflexions autour de quatre sociétés multiraciales : Burundi, Afrique du Sud, Zimbabwé , Nouvelle-Calédonie » in Afrique Contemporaine (154), 1990, pp. 35-48.

38

La partie septentrionale du Cameroun a enregistré depuis l'avènement du pluralisme politique deux guerres tribales de nature politique entre Peul ou Foulbe et Gbaya à Meiganga dans le département de la Mbéré et entre Arabes Choa et Kotoko à Kousséri dans le département du Logone et Chari. 
du phénomène ethnique. Et le présent cas reflète le type de construction ethnique faite par les élites issues du parti politique au pouvoir qui utilisent la différence culturelle comme un élément de mobilisation des populations marginalisées pour forger une conscience ethnique afin de soutenir leur pouvoir et ses ingrédients. Il s'agit bel et bien d'une ethnicité politique maîtrisée rentable à ses tenants.

Enfin, l'analyse critique doit distinguer présentement deux types de marginalisations pour éviter toute confusion de problématique : la marginalité «kirdi », cheval de bataille du groupe sympathisant dont l'action est basée sur une relation affective avec le parti au pouvoir, objet de notre préoccupation, et la marginalité de l'ensemble de la région septentrionale exprimée à travers un document intitulé : Memorandum sur les problèmes du Grand Nord $^{39}$, œuvre de certains entrepreneurs politiques en disgrâce ${ }^{40}$, lequel défraie aujourd'hui la chronique et dont les tenants cherchent son insertion comme élément éventuel du programme étatique. C'est pour dire qu'il y a deux paliers de dépendance au NordCameroun: la marginalité locale «kirdi », ancienne formule, et l'exclusion régionale, nouvelle version. Qu'il s'agisse du niveau micro « kirdi » ou macro septentrional, les deux se définissent par rapport à l'Etat, ils expriment un besoin d'Etat.

Ceci étant, Dominique Darbon souligne que «la marginalité ne s'évalue pas en termes objectifs de possibilité de participation au processus de décision mais par comparaison avec les conditions d'accès au pouvoir offertes aux autres groupes » ${ }^{41}$. Celle-ci constitue aujourd'hui le thème mobilisateur pour les investisseurs ethno-politiques tels que l'élite politique « kirdi » et autres thuriféraires des problèmes septentrionaux. Mais les pôles de son expression et de sa transformation ou de son insertion dans le discours étatique différent de la position des acteurs, pris individuellement ou en groupe, par rapport au nœud du

Voir à propos du Memorandum, la série du journal : L'œil du Sahel n91 du 28 novembre 2002, $\mathrm{n}^{\circ} 95$ du 06 janvier 2003, n 98 du 28 janvier 2003, n 104 du 01 avril 2003, n 108 du 02 mai 2003 , etc. Il convient de signaler que ce document a provoqué le déploiement sur le terrain (tournée) des élites des trois provinces septentrionales appuyées, à chaque étape, par un représentant du RDPC dont l'objectif est de démentir publiquement celui-ci et ses auteurs. Cette tournée débuta le 30 mai 2003 par Garoua, continua à Ngaoundéré le 12 juillet et se termina le 14 juillet à Maroua de la même année.

40

L'on peut citer entre autres : Daïssala Dakolé , actuel ministre d'Etat des Transports et président du MDR, parti politique de l'opposition, Moustapha Hamadou , ancien Vice-premier ministre en charge de l'Urbanisme et de l'Habitat, président de l'ANDP, parti politique de l'opposition et actuel Ministre chargé des missions à la présidence de la république, Gasagaye Antar, ancien secrétaire d'Etat en charge de l'administration pénitentiaire, président de l'UPR, parti politique de l'opposition, Tchiroma Issa, ancien ministre des Transports et membre fondateur de l'ANDP, Maïdadi Saïdou , ancien premier vice-président du SDF, principal parti politique de l'opposition et président de l'Alliance des Forces Libérales, etc. Toutes ces personnalités sont originaires de la partie septentrionale du pays.

D. Darbon, op. cit. p. 45 . 
pouvoir central. Il apparaît ainsi légitime de poser la question selon laquelle : comment apprécier la marginalité politique «kirdi»? Par rapport à qui les «kirdi» sont ou s'estiment exclus ? Si dépendance construite il y a, quels sont ses motivations ou fondements?

En fait, sans entrer dans un débat relatif à la théorie politique des phénomènes identitaires, il convient de dire tout simplement qu'à l'origine de la construction de l'ethnicité « kirdi » et celle peul connue sous le holisme d'«islamo-peul » au Nord-Cameroun, se situe la peur de la marginalisation : d'une part du côté peul, la peur de la supériorité numérique « kirdi » a conduit le régime du Président Ahidjo ${ }^{42}$ à faire de la religion musulmane une variable de mobilité et d'ascension sociale ; une ressource politique facilitant l'accès aux sites du pouvoir. C'est pourquoi certaines élites politiques « kirdi » de la première heure embrassèrent l'islam comme leur nouvelle religion ${ }^{43}$, sous réserve du droit et de la liberté du choix de sa religion. D'autres parvenaient à dénicher dans leur généalogie un ascendant imaginaire, du moins musulman sinon Peul, plus exactement à concocter un nom à consonance musulmane pour, soit maintenir leur position, soit accéder aux réseaux du pouvoir. Ce phénomène implique en même temps la volonté de domination à travers la religion musulmane et l'élargissement de « l'ethnie musulmane » pour contrer la majorité « kirdi ». D'autre part, du côté non musulman, bien que mue par les prébendes qu'offre la proximité avec l'Etat, tout porte à croire que la construction de l'ethnicité par l'élite «kirdi » trouve son fondement dans la peur d'être davantage exclue de la scène politique ; d'où leur soutien, toutes proportions gardées, systématique au régime en place.

Tous comptes faits, la marginalisation reste aujourd'hui le thème fétiche autour duquel s'articulent tous les efforts de manipulation des groupes sociaux « kirdi » par leur élite avec son aile culturelle, à savoir: la Dynamique Culturelle Kirdi dont l'activité devient très intense durant les moments électoraux où les étudiants se transforment momentanément en «mercenaires politiques » à la solde de l'élite.

Profitant de l'ampleur du chômage, de «l'avortement du processus de production par la fonction publique d'une classe moyenne » selon cette formule de Luc Sindjoun ${ }^{44}$, les étu-

Selon nos investigations, le Président Ahidjo redoutait terriblement les « kirdi » car leur éclosion certaine et inévitable allait constituer un danger réel d'asphyxie des siens.

43

Le cas le plus édifiant et typique est celui de Jean Akassou, ancien ministre, qui s'islamisa en 1978 dans le but inavoué de plaire au Prince et par ricochet de conserver son portefeuille.

44

L. Sindjoun, «Le champ social camerounais : désordre inventif, mythes simplificateurs et stabilité hégémonique de l'Etat », in Politique Africaine (62), juin 1996, p. 58. 
diants et autres « déchets sociaux ${ }^{45}$ de l'ajustement, sous l'encadrement de la «bourgeoisie kirdi », se déploient dans leurs villages respectifs à la quête des suffrages pour les investisseurs identitaires qui ne cessent de miroiter à leurs clients, soit l'accès à l'emploi, soit des retombées non négligeables sous forme monétaire, bref des avenirs meilleurs. Une question se pose ici : comment se perçoit la marginalité politique «kirdi »?

La configuration qu'a prise la « revendication kirdi » depuis un certain temps à la suite de la libéralisation de la vie politique au Cameroun ne peut se comprendre qu'en référence à un contexte idéologique et politique qui conditionne la rétribution politique dont les postes de responsabilité sont l'expression à l'apport des suffrages à une formation politique quelconque. Bref, il s'agit de la logique du donner et du recevoir.

La marginalité politique « kirdi » dont la sous-représentation politique est l'élément fondamental de définition s'exprime par une inadéquation entre le nombre de ces populations et leur poids sur l'échiquier national. On assiste ici à un cumul d'une identité contradictoire entre une majorité démographique et une minorité politique. Comparativement au groupe voisin islamo-peul par rapport auquel on mesure la marginalisation «kirdi », nul ne peut démentir la visibilité du déséquilibre. Si l'on s'en tient seulement à l'équipe gouvernementale au sens strict du terme, étalon de mesure assez suffisant pour apprécier l'exclusion des « kirdi » car les ministres relevant de ce dernier groupe ne représentent pratiquement rien par rapport au poids numérique de leur clientèle locale. Cette réalité est tellement saillante au Cameroun. Pour s'en convaincre, il faut seulement s'en tenir à l'observation de JeanMarc Ela selon laquelle «cette situation de marginalisation résulte de la manière dont l'Etat colonial et l'Etat post-colonial ont géré le pays (...). Le kirdi s'est retrouvé doublement soumis à un système de domination lamidale, ensuite coloniale et post-coloniale ${ }^{46}$.

En tout état de cause, il existe une rupture profonde aux causes multiples entre la masse « kirdi » et l'Etat central camerounais depuis le projet nationaliste pré-colonial, colonial et post-colonial. La revendication politique qu'on observe aujourd'hui, due au déséquilibre structurel qui a induit une affirmation réactive des élites puis de la masse dans une certaine mesure, est et reste politique. Ce qui veut ainsi dire que la question kirdi ne saurait plus être soustraite du débat ethnique au Cameroun sans toutefois s'apparenter dans une certaine mesure à la question bamiléké qui serait selon Dieudonné Zognong ${ }^{47}$ un projet de traduction politique du contrôle des surfaces économiques et démographiques, à la limite une

45

46

47

Par «déchets sociaux » de l'ajustement, il faut comprendre : les compressés des sociétés privatisées, les «déflatés » du secteur public, les déscolarisés résultant du désengagement de l'Etat, les migrants de retour, etc.

J.-M. Ela, in Bulletin de liaison culturelle du monde Kirdi ${ }^{\circ} 001$ de juillet 1994, p. 09 .

D. Zognong, La question Bamiléké pendant le retour de l'ouverture démocratique au Cameroun : retour d'un débat occulté. Publication du CIREPE n 1 . 
quête d'hégémonie au Cameroun selon l'imagerie populaire et même la culture savante. La question kirdi est aussi différente de la question berbère qui reste davantage linguistique ${ }^{48}$. Contrairement aux Bamiléke, les Kirdi ne disposent d'aucun pouvoir économique quelconque pour prétendre influencer le pouvoir politique suprême, ils n'ont aucune tradition mercantile encore moins une culture d'épargne. Leur situation, sans toutefois demeurer unique en son genre, reste tout de même spécifique en période de pluralisme politique où le capital humain constitue une ressource d'acquisition des positions de pouvoir.

Ceci dit, l'offre présidentielle d'une responsabilité ministérielle à une personnalité quelconque, bien que n'étant pas complètement désintéressée, n'obéit pas nécessairement à la logique de «contre-don de la reconnaissance collective traduite par un vote massif en faveur du régime " ${ }^{49}$ comme l'a constaté Luc Sindjoun. Cette observation mérite d'être relativisée ici en ce sens qu'aussi bien à l'élection présidentielle du 11 octobre 1992 que celle du 12 octobre 1997 sans oublier les scrutins municipaux et législatifs jumelés de juin 2002, l'on a enregistré un vote massif des populations non musulmanes du Nord-Cameroun en faveur du RDPC, parti au pouvoir, sans que cela n'ait donné lieu à une rétribution qui soit proportionnelle à leur enthousiasme électoral pour cette formation politique au moment de la formation des différentes équipes gouvernementales.

Aussi, la dynamique du pluralisme politique actuel fait du champ politique un marché où s'échangent des produits, notamment durant les moments électoraux où la prise de position pour un parti dans l'isoloir est susceptible de donner lieu à une récompense en termes de poste de responsabilité. Ainsi convient-il de signaler que les détenteurs des produits politiques doivent en principe les mettre en valeur et non les brader comme semblent le faire les élites, investisseurs ethno-politiques «kirdi », avec leur poids démographique, denrée très recherchée et discutée en période de concurrence politique. En d'autres termes, cela voudrait dire qu'on peut aussi monter les enchères sur le marché politique comme ce fut le cas de Dakolé Daïssala en 1992 où, avec 06 députés issus de son parti le MDR, il réussit à négocier avec le RDPC 04 postes ministériels dont celui d'Etat en charge des Postes et Télécommunications qu'il occupa lui-même.

Voir à propos : M. Cote, Le Berbère : Des langues et des territoires ; Salem Chaker, La question berbère dans le Maghreb contemporain et enfin Belkacem Lounes : Les Berbères, ou le combat d'un peuple pour sa liberté. Tous ces textes sont dans Diplomatie, Magazine, mai-juin 2003/n ${ }^{\circ}$, pp. 71-79. 


\section{Conclusion et perspectives}

Ceci étant, à la faveur de l'avènement du processus de démocratisation, l'on ne saurait encore occulter l'existence du moins d'une question si non d'un problème kirdi au Nord du Cameroun, problème que le paradigme constructiviste de l'ethnicité permet de mieux comprendre comme étant dans une bonne mesure un construit politique et/ou idéologique. Le nier serait de la mauvaise foi, l'exagérer est irresponsable comme le conseillait Dieudonné Zognong à propos de ce qu'il appelle le problème Bamiléké ${ }^{50}$.

La dynamique d'émancipation que déploient les populations non musulmanes du NordCameroun à la faveur de la ré-instauration du pluralisme politique et social est complexe. L'initiative des promoteurs de la Dynamique Culturelle Kirdi, qu'on situait au début dans une mouvance purement «intello-culturelle » sert principalement d'autres intérêts à partir du mythe identitaire servant de mobilisation et de recompositions sociales. Bref, l'ethnicité « kirdi » est instrumentalisée par son élite. Celle-ci constitue à l'heure actuelle une importante ressource de positionnement sur l'échiquier politique national.

Ainsi, il apparaît important de souligner que l'émancipation des «païens » de la partie septentrionale du Cameroun reste sur des trajectoires élitistes, sursis à l'affranchissement des couches paysannes et défavorisées des ponctions et autres brimades de la part des différentes forces rétrogrades et répressives dont regorge cette partie du territoire. Le processus de démocratisation amorcé ne semble pas encore constituer le «Printemps kirdi ». Et cette inconséquente réalité ambiante constitue un écueil à l'enracinement d'une véritable démocratie au Cameroun et fait de cette région un foyer permanent de tension que les pouvoirs publics se doivent de gérer à tout moment avec habileté pour éviter tout débordement. C'est une occasion idoine qu'il faut saisir ici pour évoquer brièvement un fait nouveau qui vient aiguiser davantage le clivage musulmans/non musulmans au Nord-Cameroun : le conflit foncier qui oppose les migrants ${ }^{51}$ à la $\mathrm{SAIB}^{52}$ dans la région de la Bénoué.

En effet, l'histoire des migrants est intiment liée au décret présidentiel nº72/332 du 17 juillet 1972 créant la Mission d'Etude et de l'Aménagement de la Vallée Supérieure de la Bénoué. Il s'agissait d'un projet visant le développement des zones rurales situées dans la région Nord-Est de la Bénoué, lesquelles étaient inhabitées et inexploitées. A cet effet, les pouvoirs publics font donc déplacer des populations de certains villages de l'Extrême-Nord avec leur force de travail pour les établir sur les terres en friche comprises entre la ville de Lagdo et celle de Bibémi. Il s'agit essentiellement des Guiziga, Maffa, Mofou, Moundang,

51 Dognong, op. cit

51 Par migrants ici, il faut entendre les populations qui ont été déplacées de leurs villages respectifs dans le cadre de la viabilisation de la vallée supérieure de la Bénoué.

52

Société Agro-Industrielle de la Bénoué. 
Toupouri, Massa, etc. Ceux-ci mirent en valeur ces superficies qui font actuellement l'objet des convoitises de certaines élites engendrant en même temps l'hostilité des autorités traditionnelles vis-à-vis des migrants considérés comme « allogènes », envahisseurs, etc.

C'est dans cette logique d'hostilité vis-à-vis des migrants fragilisés par les exactions des chefs traditionnels que survient le problème foncier qui les oppose à la SAIB. Celle-ci a introduit une demande d'obtention de titres fonciers pour arracher aux migrants un lot de terrain de 2405 hectares à Gounougou dans une banlieue de Lagdo dans le but d'y faire la culture intensive du riz. Cette demande provoqua une vive opposition des migrants qui n'entendent pas se laisser faire comme l'atteste le propos d'un des chefs des villages des migrants: «Si nous étions venus de nous-mêmes, nous ne ferions aujourd'hui aucune opposition. Mais c'est à la suite d'un décret que nous sommes installés ici. Et selon les termes de ce décret, il n'est fait mention nulle part que nous repartirions au bout d'un certain temps $»^{53}$.

L'intérêt que suscite ce qu'il est convenu d'appeler aujourd'hui affaire SAIB/Migrants ne se situe pas au niveau de l'imbroglio juridique qu'on observe entre les protagonistes, surtout autour du décret $\mathrm{n}^{\circ} 76 / 167$ du 27 avril 1976 fixant les modalités de gestion du domaine de l'Etat et le décret $n^{\circ} 76 / 165$ du 16 juillet 1976 fixant les conditions d'obtention du titre foncier mais plutôt le projet d'expropriation des migrants dans le cadre de la SAIB confirme la précarité historique des rapports entre musulmans et non musulmans au NordCameroun car tous les migrants sont « kirdi » et il semble que tous les actionnaires de ladite société industrielle sont d'obédience musulmane selon l'observation d'un fils de migrant ${ }^{54}$. D'ailleurs l'administrateur de la SAIB, Me Abdoulaye Harissou, n'y va pas par quatre chemins lorsqu'il y mêle Dakolé Daïssala, non musulman et président du MDR parti d'opposition et un des rédacteurs du Mémorandum sur les problèmes du Grand-Nord, en le qualifiant «d'aveugle tribaliste, de haine viscérale vis-à-vis des Peulhs». Il y va plus loin en faisant de la société dont il est administrateur une affaire d'Etat et fait presque une déclaration de guerre lorsqu'il dit: «En tout état de cause, il faut que tous ceux qui combattent ou veulent combattre la SAIB sachent qu'ils sont en train de jouer avec le feu (...). Nous ne nous laisserons pas faire, et ne reculerons devant aucun obstacle. Qu'on se le dise et à bon entendeur, salut ! » 55

Il est ainsi clair qu'une gestion maladroite de l'affaire SAIB/Migrants pourrait conduire à des extrémismes ethniques et à la cristallisation des clivages culturels susceptibles de faire

\footnotetext{
53 Voir L'œil du Sahel n 110 du 16 mai 2003, p. 06.

54 L'œil du Sahel n ${ }^{\circ} 116$ du 16 juillet 2003, p. 11.

55

Voir L'œil du Sahel n¹14 du 23 juin 2003, pp : 10-11.
} 
obstruction à toute entreprise de modernisation, de développement et de démocratisation de la société camerounaise.

Ce préalable évoqué, l'amalgame entre le problème historique de la domination des « kirdi » par les Peul et la nouvelle dimension de la marginalisation du Grand Nord mise en lumière par d'anciens ministres à travers le Memorandum sur les problèmes du Grand-Nord complique davantage la situation et exige de la part de la communauté « kirdi » une grande vigilance, faute de quoi celle-ci se verra une de plus exclue des préoccupations étatiques. En outre, la participation politique des «kirdi » ne doit pas être cantonnée à des trajectoires élitistes, mais populaires, c'est-à-dire que la nomination d'un «kirdi » au poste de ministre ne résout pas le problème de positionnement de toute la communauté par rapport au pouvoir. Il faut que la communauté pèse de son poids sur l'orientation et les stratégies du pouvoir central. Cela nécessite de la part des masses paysannes et populaires une certaine culture politique et une certaine culture d'organisation dont l'école institutionnelle reste l'un des pré-requis qui pourra mener le peuple «kirdi » à sa totale libération sur le triple plan politique, économique et culturel. Dans le cas contraire, les masses rurales demeureront toujours ce que la littérature politique camerounaise appelle «bétail électoral » malgré que depuis la réapparition du vote plural, elles participent et influencent parfois la structuration du jeu politique local et national.

Il y a ainsi lieu d'affirmer qu' un processus d'émancipation des masses « kirdi » est amorcée en dépit des pesanteurs historiques et les avatars de la gestion du pouvoir actuellement. Cela ne doit pas pour autant évacuer de la réflexion du champ analytique la question selon laquelle le souci d'être soi, «kirdi», d'avoir une identité politique «kirdi » pourra-t-il résister et survivre à la dynamique unificatrice de la mondialisation ? Cette interrogation reste pertinente. 